\title{
Will the leprosy endemic in Rwanda soon be under control?
}

\author{
P STES* \& X MALÂTRE $\dagger$ \\ *Damien Foundation Rwanda, BP 278 Gisenyi, Rwanda; $\dagger$ Région \\ Sanitaire Gisenyi, Rwanda
}

Accepted for publication 23 February 1989

\section{Introduction}

It appears that leprosy in Rwanda is becoming a rather rare disease. By the end of 1987, 1142 cases were still under treatment, a prevalence rate of $0 \cdot 17$ per thousand. Prevalence rates declined from 0.26 per thousand in 1982 , by an average of 0.018 per thousand a year. However, it is necessary to know whether the number of known patients reflects reality, and if case finding has been adequate. In other words: has the detection rate been a reliable indication of the incidence rate? This paper studies the problem, and tries to see if any conclusions can be made about the transmission of leprosy in the Rwandan population.

In the past, it has been suggested that a prevalence survey would be the most accurate way to evaluate leprosy prevalence. A preliminary study of the project however showed that, if we wanted to obtain acceptable margins of error, the sample to examine would be of about 225,000 persons. ${ }^{1} \mathrm{~A}$ survey of such size would be costly in time, money and personnel. Unfortunately, these disadvantages would outweigh any advantages to be gained f rom this procedure. So, we had to look for other methods to evaluate the importance of the leprosy problem in Rwanda, and particularly evaluate some epidemiological indices relating to the incidence rate.

\section{Materials and methods}

From 1964 to 1984, leprosy control in Rwanda was essentially organized by the Damien Foundation Belgium (DFB), in cooperation with the Rwandan Authorities. Detection and treatment of ambulatory patients was done by mobile units covering most of the country. In 1984, the Ministry of Health created a National Service for Leprosy Control (Service National de Lutte Contre la Lèpre, SNLCL). The SNLCL, sustained by the Damien Foundation Belgium, continued working with mobile units, insisting particularly on the integration of leprosy control with primary health care. Over 200 health centres out of 260 existing in Rwanda are visited at least 4 times a year.

Detection of new cases is done in a semipassive way-patients presenting themselves at the dispensaries or health centres visited by the mobile units. Leprosy cases are identified by the dispensary nurse, or by the person in charge of the mobile unit, during dermatology consultations. Information campaigns are being organized to inform people about the early signs of leprosy so as to motivate them to present themselves at the dermatology consultations. New patients are invited to bring along their children and household contacts for examination.

From 1977 to 1987, 564 new cases of leprosy were diagnosed and registered in Rwanda. At the 
end of each year, a statistical form was made up by the representative of the Damien Foundation Belgium, from the patients' individual forms. The data of these statistical forms are used in this paper.

From 1982 multidrug therapy (MDT) was introduced for all newly detected cases. An individual form was completed at the moment of detection for all new patients, including clinical examination in detail, skin smear results and histopathology, using the protocols elaborated by Professor Pattyn of the Antwerp Institute for Tropical Medicine. The individual forms for new cases 1982-7 have been reviewed, excluding those who died or disappeared; 233 forms out of 257 were studied and the following data were extracted:

age at detection (from identity cards);

index cases (did the patient know any leprosy patients personally?). Those with first degree family ties and husbands and wives were considered as household contacts. Most of the index cases who were household contacts and some of the other index cases were known leprosy patients under treatment;

disability grade on presentation, using the previous $0-3$ grades WHO codef or hands, feet and eyes. ${ }^{2}$

A patient was considered as disabled if he had at least one member or one eye disabled at grade 2; leprosy classification, based on clinical examination, skin smears (examined by the Service National de Lutte Contre la Lèpre) and histopathology (Professor Pattyn). A bacteriological index (BI) of

0 and 1 was classified as paucibacillary (PB), a BI of 2 or more as multibacillary (MB); sex;

number of skin lesions for PB patients. (We separated those presenting 3 skin lesions or less, and those presenting more than 3 skin lesions.)

Population figures are based on those of the 1978 census, the 1983 'Enquête National sur la Fécondité' (National Investigation on Fertility) and the estimated annual growth rate. All these figures were presented by the National Office for Population (ONAPO). ${ }^{3}$ The average number of household contacts, people living in the same house as the leprosy patients, was estimated according to a sample taken at random from 200 of the questionnaires of all known leprosy patients, established by the social workers of the Service National de Lutte Contre la Lèpre. Operational indices after detection will not be discussed in this paper.

\section{Statistics}

The differences between groups of patients were analysed using the $\chi^{2}$ test, or the $\chi^{2}$ test including Yates' correction if the number of patients was too small. Results were accepted as statistically significant if $p \leqslant 0 \cdot 05$. Graphical tendencies were evaluated by means of Spearmans' correlation coefficient of order.

\section{Results}

1 Table 1 shows the annual number of newly detected cases (NC), the number of cases under 15 years of age, the number of multibacillary cases (MB), the number of patients disabled at detection, the population covered and the detection rate (DR) per 100,000. The percentages are given in brackets for the different data.

2 The distribution by age and by year of detection of new patients $1982-87$ is shown in Table 2 . The distribution by age and sex of new cases 1982-7 is represented in Table 3 together with the number of multibacillary cases (MB), the number of patients disabled at detection, the number of patients with an index case and the number of patients whose index case was a household contact. The data of Tables 2 and 3 were collected from 233 individual forms. 
Table 1. New cases registered and detection rate in Rwanda 1977-87

\begin{tabular}{lrrlrrrrr}
\hline Year & NC & Age & $0-14(\%)$ & MB & $(\%)$ & Disabled (\%) & Population & $\begin{array}{l}\text { DR per } \\
100,000\end{array}$ \\
\hline 1977 & 56 & 4 & $(7 \cdot 2)$ & 28 & $(50 \cdot 0)$ & & $4,645,822$ & $1 \cdot 2$ \\
1978 & 81 & 10 & $(12 \cdot 3)$ & 40 & $(49 \cdot 4)$ & & $4,819,317$ & $1 \cdot 7$ \\
1979 & 49 & 4 & $(8 \cdot 2)$ & 21 & $(42 \cdot 8)$ & & $4,992,812$ & $1 \cdot 0$ \\
1980 & 57 & 2 & $(3 \cdot 5)$ & 33 & $(57 \cdot 8)$ & & $5,172,554$ & $1 \cdot 1$ \\
1981 & 64 & 4 & $(6 \cdot 3)$ & 24 & $(37 \cdot 5)$ & & $5,358,766$ & $1 \cdot 2$ \\
1982 & 52 & 4 & $(7 \cdot 6)$ & 13 & $(25 \cdot 0)$ & $16(30 \cdot 7)$ & $5,542,042$ & $0 \cdot 9$ \\
1983 & 55 & 2 & $(3 \cdot 6)$ & 15 & $(27 \cdot 3)$ & $11(20 \cdot 0)$ & $5,739,061$ & $1 \cdot 0$ \\
1984 & 56 & 1 & $(2 \cdot 0)$ & 16 & $(28 \cdot 5)$ & $17(30 \cdot 3)$ & $5,943,085$ & $0 \cdot 9$ \\
1985 & 25 & 5 & $(20 \cdot 0)$ & 7 & $(28 \cdot 0)$ & $8(32 \cdot 0)$ & $6,154,362$ & $0 \cdot 4$ \\
1986 & 25 & 3 & $(12 \cdot 0)$ & 7 & $(28 \cdot 0)$ & $4(16 \cdot 0)$ & $6,373,591$ & $0 \cdot 4$ \\
1987 & 44 & 4 & $(9 \cdot 1)$ & 14 & $(31 \cdot 8)$ & $9(20 \cdot 5)$ & $6,603,040$ & $0 \cdot 7$ \\
\hline Total: & 564 & 43 & $(7 \cdot 6)$ & 218 & $(38 \cdot 7)$ & $65(25 \cdot 3)$ & & \\
\hline
\end{tabular}

Table 2. Distribution by age and by year of detection of new patients in Rwanda 1982-7

\begin{tabular}{lrrrrrr}
\hline Age & $0-14$ & $15-29$ & $30-44$ & $45-59$ & $60+$ & $?$ \\
\hline 1982 & 4 & 11 & 14 & 12 & 6 & \\
1983 & 2 & 10 & 17 & 14 & 5 & 1 \\
1984 & 1 & 13 & 17 & 14 & 6 & \\
1985 & 3 & 5 & 8 & 6 & 2 & \\
1986 & 4 & 3 & 7 & 5 & 4 & \\
1987 & 3 & 8 & 10 & 9 & 7 & 2 \\
\hline Total & 17 & 50 & 73 & 60 & 30 & 3 \\
\hline
\end{tabular}

Table 3. Distribution by age and sex of new patients in Rwanda 1982-7

\begin{tabular}{lrrrrrrr}
\hline Age & $0-14$ & $15-29$ & $30-44$ & $45-59$ & $60+$ & $?$ & Total \\
\hline New patients 1982-7 & 17 & 50 & 73 & 60 & 30 & 3 & 233 \\
Males & 11 & 26 & 36 & 27 & 16 & 2 & 118 \\
Females & 6 & 24 & 43 & 28 & 14 & 1 & 115 \\
MB & 5 & 24 & 22 & 12 & 5 & 1 & 69 \\
Disabled at detection & 1 & 10 & 22 & 17 & 13 & 2 & 65 \\
Patients with index cases & 17 & 18 & 30 & 22 & 6 & - & 93 \\
Household contact index cases & 15 & 12 & 13 & 6 & 3 & - & 49 \\
\hline
\end{tabular}

In 3 cases (adults), age was not marked on the patients' individual form. Out of the male patients, 39 were $\mathrm{MB}$, and 31 were disabled at detection. Out of the female patients, 30 were $\mathrm{MB}$ and 34 disabled. Eight out of 49 patients with household contact index cases were disabled at detection. 3 The number of paucibacillary (PB) cases showing 3 skin lesions or less at detection was 26 out of 164 PB cases. Out of 26, 2 were disabled, 14 were male and 12 female. The distribution according to age groups is shown in Table 4. 
Table 4. Age distribution of new PB patients presenting 3 skin lesions or less at detection, Rwanda 1982-7

\begin{tabular}{lrrrrrr} 
Age & $0-14$ & $15-29$ & $30-44$ & $45-59$ & $60+$ & total \\
\hline New PB patients 1982-87 & 12 & 26 & 51 & 48 & 25 & 164 \\
Patients with 3 skin lesions or less & 2 & 3 & 9 & 8 & 4 & 26 \\
\hline
\end{tabular}

\section{Discussion}

1 If we want to study the incidence of leprosy in a given population, the first index to look at is the detection rate, which is represented in Table 1 and Figure 1. Since 1977, this rate shows a general decrease. This tendency was tested by means of Spearmans' correlation coefficient of order, which is $-0.891(d l=9)$. The test gives a statistically significant result $(p \leqslant 0 \cdot 01)$.

Some of the annual detection rate figures need further comment. The steep dip during 1985-6, the years of transition, may be due to diminished control activities while the Service National de Lutte Contre la Lèpre was being set up. The 1987 rise could then be seen as a 'catching-up' manoeuvre of previously undetected cases, and is probably a good sign.

How can we ascertain whether the detection rate is representative of the incidence rate? We used three indices for this:

1.1 The most reliable index to estimate the quality of detection is to follow the proportion of disabled patients among newly detected cases. Indeed, if patients are detected at an early stage of disease, they are not yet disabled. In the best projects for leprosy control, the proportion of disabled patients is about $5-8 \%$ of the new patients. ${ }^{4}$ For projects having a higher proportion of disabled new cases, a gradual decrease of this percentage means that detection is improving.

For Rwanda, reliable figures can be found from 1982 onwards. (Table 1). In total, 65 of 257 new cases detected since 1982 were disabled, or $25.3 \%$. This is a relatively high percentage, compared with other countries and services. The evolution of the proportion of disabled new cases is represented in Figure 2. There is no significant decrease of the proportion. (Spearmans' test $r^{\prime}=0 \cdot 31, d l=4$, nonsignificant result.)

1.2 The second index we studied is the proportion of new PB patients showing 3 skin lesions or less at detection. Among these patients are those who presented themselves at the first sign of the



Figure 1. Evolution of detection rate per 100,000, Rwanda 1977-87. 


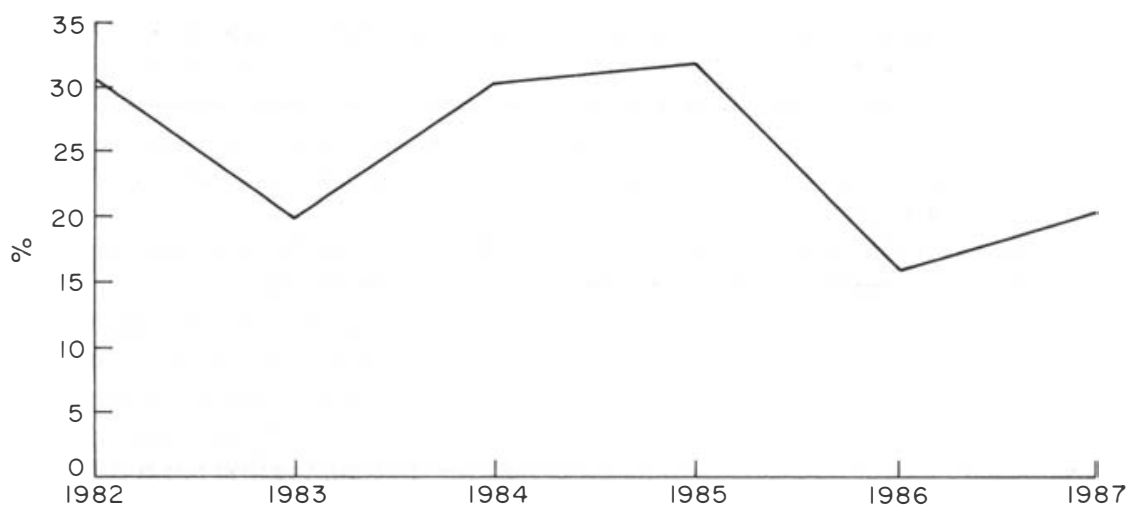

Figure 2. Evolution of the percentage of new cases disabled a t detection, Rwanda 1982-7.

disease, and were also recognized at an early stage. We chose this index according to the study of new therapeutic schemes of Professor Pattyn of the Antwerp Institute for Tropical Medicine, in which we collaborated, that shows significantly better therapeutic results for patients showing 3 skin lesions or less. ${ }^{5}$ If we study the new patients $1982-7$, we find significantly less disabled patients among those presenting 3 skin lesions or less, than among the PB patients presenting more than 3 skin lesions ( 2 out of $26 v s 47$ out of $138, \chi^{2}$ (Yates) $=6 \cdot 055,0 \cdot 02>p>0 \cdot 01$ ). These statistics confirm the assumption that detection is particularly early in the group of patients presenting 3 skin lesions or less. Out of 164 new PB cases 1982-7, 26 or 16\% presented 3 skin lesions or less at detection. Even if it is difficult to compare these figures with other leprosy control centres or countries, it is obvious that this percentage is not high.

1.3 The third index is the evolution of the proportion of MB cases to the total of newly detected cases. At the start of a leprosy control programme, this proportion is elevated. When the programme is under way, the proportion gradually decreases, and stabilizes when the detection rate becomes close to the true incidence. If early detection is taking place, the proportion should be constant too. ${ }^{6}$

For Rwanda, the proportion of $\mathrm{MB}$ cases to the total of new cases is represented in Table 1 and Figure 3. This proportion shows an important decrease, coming from $\pm 50 \%$ before 1981 to $25 \%$ after 1982. During the period 1982-7, the proportion stabilizes between 25 and $31 \%$. (Spearmans' test $r^{\prime}=0 \cdot 743, d l=4$, not significant).

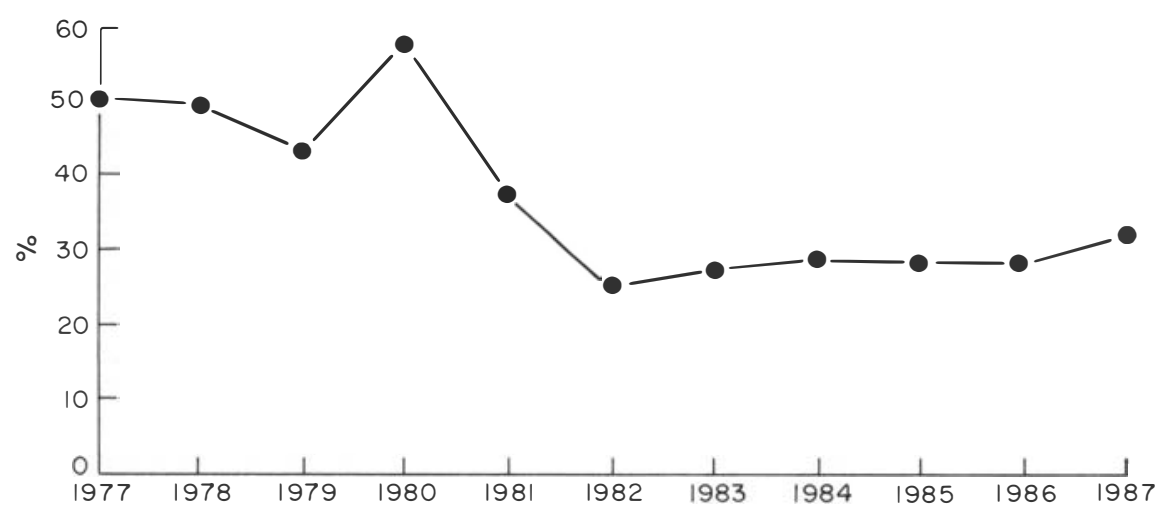

Figure 3. Evolution of the percentage of new MB cases among the total of new cases, Rwanda, $1977-87$. 
Here, it is important to watch more closely the diagnostic tools used during the 1977-81 period versus those used in 1982-7. Before 1981, diagnosis and classification of patients was essentially based on clinical aspects: skin smears and biopsies were not systematically performed. After 1982, every new patient had at least one skin smear and one skin biopsy taken. Obviously, diagnoses made from 1982 onwards were much more reliable, and it is possible that MB cases were overdiagnosed during the 1977-81 period.

The stabilization of the proportion of the new MB cases to the total of new cases after 1982 seems to indicate that detection rate approaches the true incidence rate.

When we look at the first two indices of quality of detection, we have to conclude that detection of cases in Rwanda is certainly not very early. This implies that probably a number of cases are not detected. However, it is impossible to determine more accurately the number of patients.

The evolution of the proportion of new MB cases to the total of new cases seems to be in contradiction with the first two indices, indicating that the detection rate is not too far away from the true incidence rate. It may be that, although patients are obviously not detected in the early stage, most are actually detected later on, the time lapse between the appearance of the first signs and detection being long but fairly constant. The evolution of the detection rate therefore is a correct indication of the evolution of the incidence rate, and the underestimation of the incidence rate is constant, not detecting the one-macule cases, as they often heal spontaneously.

The causes of overall late detection are complex and will not be discussed in detail here. We want to mention however three reasons, which we feel are important: as the disease is becoming rare, early signs are not easily recognized by the population or by medical workers; leprosy still carries an important social stigma; and in Rwanda, detection seems to be generally late for most diseases and leprosy is no exception.

2 After discussion of the detection rate, a second criterion studied is the distribution of the new cases by age and sex. This results in important information regarding endemicity of the disease and its transmission in a population.

2.1 The distribution of new cases according to age groups. This distribution is very similar in many high endemic areas, where there is a clear peak at ages 10-14 followed by a depression which in turn is followed by a rise and a plateau covering $30-60 .^{7}$ If there is a peak in the adult ages, and the detection rate is low or becomes minimal among children and young people, one can expect an extinction of the leprosy endemic. ${ }^{8} \mathrm{Li}$ et al. demonstrated a gradual shift towards older age groups in Shandong, China, over a 25 -year period when incidence rates were declining. ${ }^{9}$

In $\mathrm{R}$ wanda, the period of time for which we have reliable figures regarding age distribution and the numbers of patients are too limited to show any significant evolution during that period. Age distribution over the years 1982-7 show the same characteristics and therefore all new patients were seen as one single patient group.

Table 5 represents age distribution of the new cases 1982-7, as drawn from the individual patients' forms, including the relative percentage of each group in the total of new cases. The percentage of the total population belonging to each age group, the average number of people for the 1982-7 period in each age group and the average annual age-specific detection rate (1982-7) per 100,000 are shown.

The average annual age-specific detection rate (1982-7) is represented graphically in Figure 4. The relative risk for each age group and its confidence interval are represented in Table 6 . The relative risk is the risk for any individual of a certain age group to be detected, in comparison with the other age groups.

Looking at these figures, however, we should ask ourselves whether they reflect reality: perhaps detection among children is of very poor quality? To answer this question, we wanted to compare the indices for quality of detection for the different age groups. But, as children are known to develop a generally benign form of leprosy leading to few disabilities, ${ }^{10}$ the first index is of little value. Even if we find statistically less disabled patients among children (as is the case in Rwanda, $p$ $\leqslant 0.05$ ), this is exactly what we should expect to find, even if detection is not early at all. As for the 
Table 5. Average annual age-specific detection rate per 100,000 Rwanda 1982-7

\begin{tabular}{|c|c|c|c|c|c|}
\hline Age & $0-14$ & $15-29$ & $30-44$ & $45-60$ & $60+$ \\
\hline New patients & 17 & 50 & 73 & 60 & 30 \\
\hline$\%$ of new patients & $7 \cdot 4$ & $21 \cdot 7$ & $31 \cdot 7$ & $26 \cdot 1$ & $13 \cdot 0$ \\
\hline$\%$ of population & $48 \cdot 4$ & $25 \cdot 9$ & $12 \cdot 6$ & $8 \cdot 3$ & $4 \cdot 8$ \\
\hline Average population & $2,932,651$ & $1,569,332$ & 763,459 & 502,913 & 290,841 \\
\hline $\begin{array}{l}\text { Average annual detection } \\
\text { rate per } 100,000\end{array}$ & $0 \cdot 1$ & 0.5 & $1 \cdot 6$ & $2 \cdot 0$ & $1 \cdot 7$ \\
\hline
\end{tabular}

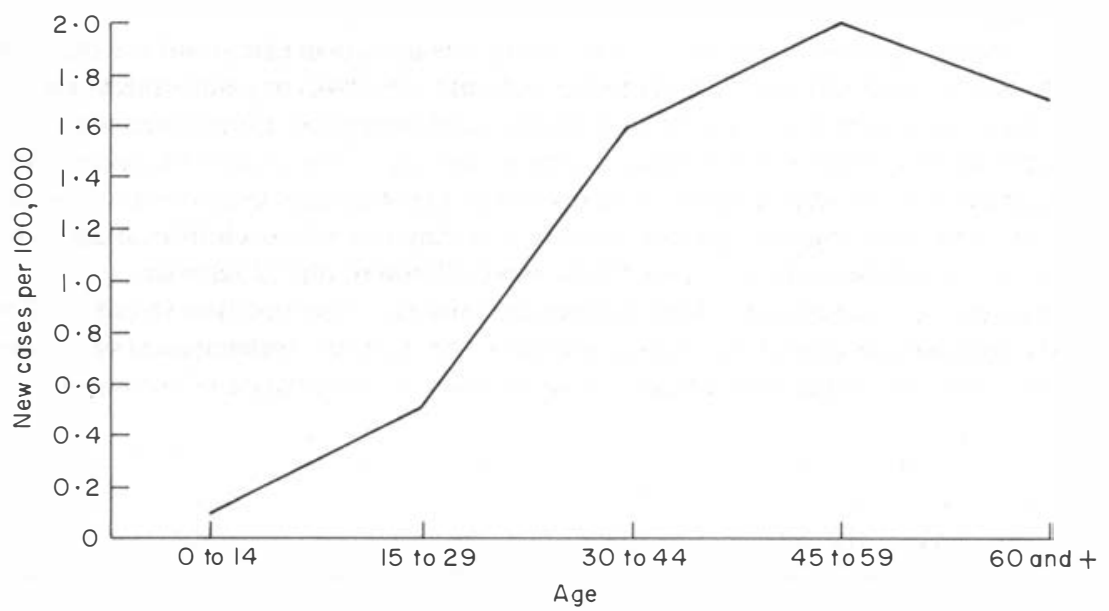

Figure 4. Average annual age-specific detection rate per 100,000, Rwanda 1982-87.

Table 6. Relative risk for different age groups, new cases in Rwanda 1982-7

\begin{tabular}{lcc}
\hline Age & Relative risk & Confidence interval \\
\hline $0-14$ & $0 \cdot 09$ & $(0 \cdot 05-0 \cdot 14)$ \\
$15-29$ & $0 \cdot 80$ & $(0 \cdot 58-1 \cdot 09)$ \\
$30-44$ & $3 \cdot 23$ & $(2 \cdot 45-4 \cdot 25)$ \\
$45-59$ & $3 \cdot 90$ & $(2 \cdot 90-5 \cdot 22)$ \\
$60+$ & $2 \cdot 98$ & $(2 \cdot 03-4 \cdot 35)$ \\
\hline
\end{tabular}

number of new cases presenting 3 skin lesions or less at the moment of detection, there was no significant difference between age groups. We can only conclude from this that we see no reason why detection should be particularly late among children.

As the overall detection rate among children and young adults is indeed very low, the age distribution of new cases in Rwanda shows the characteristics of a region where there is extinction of the leprosy endemic.

2.2 The sex distribution of new cases 1982-7 is represented in Table 3. Before 1982, no reliable figures are available. The 1982-7 figures do not show any statistically significant differences in 
detection rates for male and female, in proportion of $\mathrm{MB}$ cases for male and female, or in the indices of quality of detection.

As sex distribution for leprosy seems to vary a lot world-wide, we prefer not to makeconclusions regarding these findings.

3 Finally, it is interesting to study more closely the group of household contacts of the leprosy patients, as the occurrence of leprosy in family clusters has been particularly observed in low endemic areas. ${ }^{1}$

The Rwandan leprosy patient has a n estimated average number of household contacts of 4.6. For 1984, the 1626 known patients under treatment or under observation had 7480 household contacts. Out of these, 49 were found to have developed leprosy over a period of 6 years. The relative risk of household contacts versus the total population minus the household contacts, is extremely high: $216 \cdot 88(158 \cdot 31-296 \cdot 31)$.

Detection of new cases among household contacts was not particularly early: 8 out of 49 were disabled at detection, versus 57 out of 208 patients without index cases or whose index case was not a household contact $\left(\chi^{2}=2 \cdot 58 p \leqslant 0 \cdot 20\right.$, not significant). Eight out of 32 PB patients with household contact index cases had 3 skin lesions or less, versus 18 out of 132 other new PB patients $\left(\chi^{2}=2 \cdot 50\right.$ $p \leqslant 0 \cdot 20$, not significant). The assumption that leprosy patients play an important role as detection agents, was not confirmed. Sex distribution among new cases with household contact index cases was the same as for the total of new cases. There were 27 males and 22 females.

The elevated relative risk of household contacts and the fact observed that 15 out of 17 infected children had index cases who were household contacts, indicate that transmission of the disease in recent years has been, to a high degree, limited to the household contacts of the leprosy patients.

\section{Conclusion}

The three criteria studied: the detection/incidence rate, the age and sex distribution of new cases and the new cases among household contacts of leprosy patients, suggest that leprosy transmission in $\mathrm{R}$ wanda is low-certainly outside the family clusters - and still diminishing. Taking into account the decreasing prevalence rates, we believe that the leprosy endemic in Rwanda can be controlled within a limited period of time, certainly before the goal of the World Health Organisation for leprosy control worldwide by the year 2000 .

As to our own strategy in the field, we conclude that there should be more emphasis on contact survey and on informing the public and the medical staff of the first signs of the disease so as to make early detection easier. In this way, we hope to prevent more patients from becoming disabled.

\section{References}

1 Sundaresan TK. Les sondages dans lutte contre la lèpre-Manuel préparatoire. OMS. (WHO.CDS.LEP.86.1)

2 OMS: Directives concernant la lutte contre la lèpre, p. 198. WHO, Geneva 1985. Referring to: WHO Technical Report Series No. 459. WHO, Geneva 1970.

3 ONAPO: Rwanda 1983-Enquête National sur la Fécondité. Vol. 1 analyse des résultats, pp. 34-8. Office National de la Population, Kigali 1983.

4 Pattyn SR, Groenen G, Bourland J, Grillone S, Janssens L. A controlled therapeutic trial in paucibacillary leprosy comparing a single dose of rif ampicin followed by 1 year of daily dapsone with 10 weekly doses of rifampicin. Lepr Rev, 1987; 58: 349-58.

5 Pattyn SR, Dockx P, Cap JA. Lèpre. Microbiologie, diagnostic, traitement et lutte, p. 87. Masson, Paris 1981.

6 Hastings RC. Leprosy, p. 263. Churchill Livingstone, Edinburgh 1985.

7 Hastings RC. Leprosy, pp. 17-18. Churchill Livingstone, Edinburgh 1985.

8 Pattyn SR, Dockx P, Cap JA. La Lèpre. Microbiologie, diagnostic, traitement et lutte, pp. 87-8. Masson, Paris 1981.

9 Li et al: Leprosy Control in Shandong, China. Int J Lepr, 1985; 53, 1: 83-5.

${ }^{10}$ Languillon J: Précis de léprologie, pp. 224-5. Masson, Paris 1986.

1 Hastings RC: Leprosy, p. 20. Churchill Livingstone, Edinburgh 1985. 